\title{
Treinamento em circuito melhora cognição, funcionalidade e qualidade de vida de
}

\section{idosos}

\author{
Circuit training improves cognition, functionality and quality of life of the elderly
}

El entrenamiento en circuitos mejora la cognición, la funcionalidad y la calidad de vida de las

personas mayores

Recebido: 11/06/2021 | Revisado: 09/07/2021 | Aceito: 12/07/2021 | Publicado: 22/07/2021

Luana Otone Costa Santos

ORCID: https://orcid.org/0000-0003-2632-4727

Universidade Federal dos Vales do Jequitinhonha e Mucuri, Brasil

E-mail: luana_otoni@hotmail.com

Jessica de Oliveira Moreira

ORCID: https://orcid.org/0000-0001-5084-8200

Universidade Federal dos Vales do Jequitinhonha e Mucuri, Brasil

E-mail: jessica-dtna@hotmail.com

Mayara Marylin de Azevedo Silveira

ORCID: https://orcid.org/0000-0001-7828-5825

Universidade Federal dos Vales do Jequitinhonha e Mucuri, Brasil

E-mail: mayaramas@hotmail.com

Neumir Sales de Lima

ORCID: https://orcid.org/0000-0002-6588-2177

Universidade Federal dos Vales do Jequitinhonha e Mucuri, Brasil

E-mail: neumir@yahoo.com.br

Caíque Olegário Diniz e Magalhães

ORCID: https://orcid.org/0000-0001-7440-5463

Universidade Federal dos Vales do Jequitinhonha e Mucuri, Brasil

E-mail: caique-959@hotmail.com

Bruno Ferreira Mendes

ORCID: https://orcid.org/0000-0001-7904-4193

Universidade Federal dos Vales do Jequitinhonha e Mucuri, Brasil

E-mail: brunofmendes2009@hotmail.com

Ricardo Cardoso Cassilhas

ORCID: https://orcid.org/0000-0001-6970-2551

Universidade Federal dos Vales do Jequitinhonha e Mucuri, Brasil

E-mail: rcassilhas@yahoo.com.br

\begin{abstract}
Resumo
Introdução: O envelhecimento vem aumentando ao longo das últimas décadas, com isso se aumenta o risco de declínio cognitivo, diminuição funcional e piora da qualidade de vida. A prática de exercício físico ganha destaque por seu caráter preventivo e/ou terapêutico, de custo baixo e acessível. A maioria dos estudos utilizam períodos longos de intervenção (6 meses ou mais), com exercícios aeróbios ou resistidos, no entanto são escassos os trabalhos que utilizam da metodologia de treinamento em circuito. O presente estudo avaliou os efeitos de 12 semanas de um programa de treinamento em circuito sobre aspectos funcionais, funções cognitivas e qualidade de vida de idosos. Metodologia: Vinte idosos foram submetidos a um programa de treinamento em circuito por um período de 12 semanas e avaliados no período pré e pós intervenção. Foram utilizados: questionário SF-36, Mini Exame do Estado Mental (MEEM), Time Get up and go (TUG), Teste de sentar e levantar, Teste de flexão de antebraço, Teste de velocidade de marcha de 6 minutos. Resultados: Os dados obtidos mostraram uma melhora de $26 \%$ na média do SF-36, 7\% na velocidade da marcha para o teste de 6 minutos, e aumento de 1,6 pontos para o mini mental. Conclusão: $\mathrm{O}$ treinamento físico em circuito foi capaz de melhorar qualidade de vida, funcionalidade e cognição em idosos.
\end{abstract}

Palavras-chave: Envelhecimento; Testes cognitivos; Exercício físico.

\begin{abstract}
Introduction: Aging has been increasing over the last decades, thus increasing the risk of cognitive decline, functional decrease and worsening of quality of life. The practice of physical exercise gains prominence for its preventive and/or therapeutic character, of low and affordable cost. Most studies use long periods of intervention (6 months or more), with aerobic or resistance exercises, however there are few studies that use the circuit training methodology. The present study evaluated the effects of 12 weeks of a circuit training program on functional aspects, cognitive functions and quality of life of the elderly. Methodology: Twenty elderly were submitted to a circuit training program for a
\end{abstract}


period of 12 weeks and evaluated in the pre- and post-intervention period. The following were used: SF-36 questionnaire, Mini Mental State Examination (MMSE), Time Get up and go (TUG), Stand Lift Test, Forearm Flexion Test, 6-minute Gait Speed Test. Results: The data obtained showed a 26\% improvement in the mean of the SF-36, 7\% in the gait speed for the 6-minute test, and an increase of 1.6 points for the mini mental. Conclusion: Physical training in circuit was able to improve quality of life, functionality and cognition in the elderly.

Keywords: Aging; Cognitive tests; Exercise.

\section{Resumen}

Introducción: El envejecimiento ha ido en aumento en las últimas décadas, aumentando así el riesgo de deterioro cognitivo, disminución funcional y empeoramiento de la calidad de vida. La práctica del ejercicio físico cobra protagonismo por su carácter preventivo y/o terapéutico, de bajo y asequible costo. La mayoría de los estudios utilizan largos períodos de intervención (6 meses o más), con ejercicios aeróbicos o de resistencia, sin embargo, hay pocos estudios que utilicen la metodología de entrenamiento del circuito. El presente estudio evaluó los efectos de 12 semanas de un programa de entrenamiento en circuito sobre aspectos funcionales, funciones cognitivas y calidad de vida de los ancianos. Metodología: Veinte ancianos fueron sometidos a un programa de entrenamiento en circuito por un período de 12 semanas y evaluados en el período pre y post-intervención. Se utilizaron los siguientes: cuestionario SF-36, Mini Examen del Estado Mental (MMSE), Time Get up and go (TUG), Sitand LiftTest, Forearm Flexion Test, 6-minute Gait Speed Test. Resultados: Los datos obtenidos mostraron una mejoría del $26 \%$ en la media del SF-36, del $7 \%$ en la velocidad de la marcha para la prueba de 6 minutos, y un aumento de 1,6 puntos para la mini mental. Conclusión: El entrenamiento físico en circuito fue capaz de mejorar la calidad de vida, la funcionalidad y la cognición en los ancianos.

Palabras clave: Envejecimiento; Pruebas cognitivas; Ejercicio.

\section{Introdução}

Segundo a Organização Mundial de Saúde (OMS) define-se como idoso o indivíduo que tenha idade igual ou superior a 60 anos. Em todo o mundo, o número de idosos tem crescido de forma exponencial, e no Brasil constata-se o mesmo crescimento; em 2017 a marca de 30,2 milhões de idosos foi superada (IBGE, 2018).

Esse aumento no número de idosos é de extrema importância no que diz respeito a políticas de saúde pública para esta faixa etária, uma vez que o envelhecimento é acompanhado pelo aumento de custos diretos e indiretos de assistência médica associados ao envelhecimento da população, sobretudo quando não são adotadas medidas preventivas ou mesmo não farmacológicas com o (Lacroix et al., 2016) intuito da promoção da saúde (Copeland et al., 2018).O envelhecimento é um processo gradual, universal e irreversível, que está associado ao declínio progressivo da saúde física e mental, o que aumenta o risco de incapacidade e dependência, bem como ao aumento do número de comorbidades e redução das capacidades fisiológicas (respiratória e circulatória) (Pietta-Dias et al., 2019). Com o aumento da idade associada ao sedentarismo, processos degenerativos no sistema nervoso central, ocorrem de forma acentuada e resultam em comprometimento do equilíbrio, da força muscular e desempenho energético. Tendo em vista esse quadro, o exercício físico é apontado como um fator de prevenção e tratamento de diversas doenças que tendem a surgir com o processo de envelhecimento (Brandão et al., 2018; Lopez et al., 2018; Raso et al., 2016).

A literatura evidencia que programas de exercícios físicos em idosos devem incluir treinamento aeróbico, resistido, flexibilidade e equilíbrio. Cada tipo de exercício pode beneficiar diferentes parâmetros relacionados à saúde, mas a combinação de todos se mostra eficaz na população idosa, tendo em vista que ele pode ser usado como estratégia preventiva ou terapêutica (Cassilhas et al., 2007, 2010)além de pode promover sentimentos de bem-estar e melhora a qualidade de vida, estando associado a um menor risco de declínio cognitivo e demência (Galloza et al., 2017).

Apesar do considerável número de estudos que apontam para os benefícios do exercício físico para os idosos, a maioria dos trabalhos utilizaram de períodos prolongados de tempo (6 meses ou mais), valendo-se ou de exercício aeróbios ou resistidos, sendo raras as intervenções em modelo de circuito, assim como são escassos os trabalhos que buscam realizar a associação entre o exercício físico e atividades lúdicas. 
Sendo interessante observar que a associação entre atividades lúdicas e exercícios físicos pode ser interessante, pois podem gerar benefícios fisiológicos e psicológicos que podem agir com efeito associado de forma benéfica. Dessa maneira o presente estudo verificou os efeitos de 12 semanas de intervenção por meio de treinamento em circuito na cognição, funcionalidade e qualidade de vida de idosos. Tendo como hipótese que esses parâmetros serão melhorados.

\section{Metodologia}

Foi realizado um estudo experimental com amostragem por conveniência, em um método qualitativo (Pereira et al., 2018). A população do estudo foi constituída por 20 idosos (60 anos ou mais) de ambos os sexos, integrantes do grupo de práticas corporais da cidade de Felício dos Santos/MG.

\section{Aspectos éticos}

Este estudo foi aprovado ao Comitê de Ética em Pesquisa da Universidade Federal dos Vales do Jequitinhonha e Mucuri (UFVJM), com o número do parecer 1.099.687, e certificação 44546215.6.0000.5108. Todos os voluntários foram informados sobre os procedimentos que seriam submetidos e toda a metodologia do estudo foi explicada. Para o início do estudo, todos assinaram um termo de consentimento livre e esclarecido (TCLE), e aqueles que não sabiam assinar, preencheram com a impressão digital. O estudo atendeu aos preceitos éticos para pesquisas envolvendo seres humanos preconizados na Resolução 466/12 CNS. O estudo adotou os seguintes critérios de inclusão/ exclusão:

* Critérios de inclusão: Ter idade acima de 60 anos; ser integrante do grupo de recreação da prefeitura local; assinar o termo de consentimento livre esclarecido.

* Critérios de exclusão: Histórico de doenças cardiovasculares com sequelas que comprometam a prática regular de atividade física (hemiplegia ou hemiparesia decorrentes de acidente vascular encefálico, infarto agudo do miocárdio); demência ou outra doença neurodegenerativa; histórico de labirintite, vertigem e distúrbios de equilíbrio.

\section{Treinamento em circuito}

O treinamento em circuito foi realizado três vezes por semana (segunda-feira, quarta-feira e sexta-feira), com sessões diárias de 1hora, pelo período de três meses. Cada sessão de exercícios consistiu em:

a) Realização de jogos e exercícios lúdicos/ alongamentos (25min);

b) Realização de estações de exercícios funcionais em forma de circuito alternado por segmentos envolvendo os grandes grupos musculares, somente com o peso corporal (30min);

c) Relaxamento (5min).

A progressão do treinamento foi realizada de acordo com a tabela 1.

A Tabela 1 demonstra como se deu a progressão do exercício físico durante as 12 semanas de intervenção. 
Tabela 1. Progressão do treinamento. *Intensidade: Percepção Subjetiva do Esforço - PSE - Escala de Borg (adaptada) 0-10 (Borg, 1998).

\begin{tabular}{|c|c|c|c|c|}
\hline Mês de intervenção & Passagens & Estímulo & Pausa & Intensidade \\
\hline \multirow[t]{2}{*}{$1^{\circ}$ Mês } & $1^{\mathrm{a}}$ & $40 ”$ & $20 ”$ & PSE - 6 \\
\hline & $2^{a}$ & $30 "$ & $30 "$ & \\
\hline \multirow[t]{2}{*}{$2^{\circ}$ Mês } & $1^{\mathrm{a}}$ & $50 "$ & $20 "$ & $\mathrm{PSE}-7$ \\
\hline & $2^{\mathrm{a}}$ & $40 "$ & $30 "$ & \\
\hline \multirow[t]{2}{*}{$3^{o}$ Mês } & $1^{\mathrm{a}}$ & $60 "$ & $30 "$ & $\mathrm{PSE}-8$ \\
\hline & $2^{\mathrm{a}}$ & $50 "$ & $40 "$ & \\
\hline
\end{tabular}

Fonte: Autores.

\section{Mensuração da Massa corporal e IMC}

O peso e a estatura foram medidos em uma balança com estadiômetro acoplado (marca Welmy). O Índice de Massa Corporal (IMC) foi calculado a partir da seguinte equação: massa corporal (em kg) dividido pela estatura ao quadrado (m2).

\section{Avaliação da Qualidade de vida}

A avaliação da qualidade de vida foi realizada com a utilização do questionário SF-36 (Medical Outcomes Study 36 Item Short - Form Health Survey). Consiste em um questionário multidimensional formado por 36 itens, englobados em 8 escalas ou domínios, que são capacidade funcional (CF), Limitações por aspectos físicos (LAF), dor, estado geral da saúde (ESG), vitalidade (V), aspectos sociais (AS), aspectos emocionais (AE) e saúde mental (SM). Apresenta um escore final de 0 (zero) á 100 (obtido por meio de cálculo do Raw Scale), onde o zero corresponde ao pior estado geral de saúde e o 100 corresponde ao melhor estado de saúde (Campolina et al., 2011).

\section{Avaliação da cognição}

A cognição foi avaliada utilizando o Mini Exame do Estado Mental (MEEM). Ele fornece informações sobre diferentes parâmetros cognitivos, contendo questões agrupadas em dez categorias, cada uma delas planejada com o objetivo de avaliar "funções" cognitivas específicas possuindo um número máximo de pontuação, como a orientação temporal Espacial (10 pontos), registros (3 pontos), atenção e cálculo (5 pontos), lembrança ou memória de evocação (5 pontos), linguagem (9 pontos). O escore do MEEM pode variar de um mínimo de 0 pontos, o qual indica o maior grau de comprometimento cognitivo dos indivíduos, até um total máximo de 30 pontos, o qual, por sua vez, corresponde a melhor capacidade cognitiva (de Melo \& Barbosa, 2015).

\section{Avaliação da funcionalidade}

\section{Time Get up and go (TUG)}

O teste consiste em levantar-se de uma cadeira, sem ajuda dos braços, andar a uma distância de três metros, dar a volta e retornar. No início do teste, o participante deve estar com o dorso apoiado no encosto da cadeira e, ao final, deve encostar novamente. O paciente deve receber a instrução "vá" para realizar o teste e o tempo será cronometrado com a partir da voz de comando até o momento em que ele apoie novamente o dorso no encosto da cadeira. O teste deve ser realizado uma vez para familiarização e uma segunda vez para tomada do tempo. Pontuação: a) menos de 20 segundos para realização, 
correspondendo a baixo risco para quedas; b) de 20 a 29 segundos, a médio risco para quedas; c) 30 segundos ou mais, a alto risco para quedas (Bohannon \& Schaubert, 2006).

\section{Teste de sentar e levantar}

O participante deverá sentar-se na cadeira, mantendo os pés afastados a largura de seus ombros, sendo que seus membros superiores devem se manter cruzados ao peitoral. O avaliado deve se elevar de forma a se manter ereto e retornar novamente a posição inicial por 30 segundos. O orientador deve demostrar, logo após o participante deve repassar para depois ser avaliado. Por razões de segurança, a cadeira deve ser colocada contra uma parede, ou estabilizada de qualquer outro modo, evitando que se mova durante o teste. Pontuação: O número máximo de execuções realizadas nos 30 segundos (Lira et al., 2000).

\section{Teste de flexão de antebraço}

O participante deverá sentar-se na cadeira, mantendo os pés bem apoiados no solo, deve-se segurar o haltere com a mão dominante, sendo que o antebraço deve estar ao lado da cadeira, perpendicular ao solo. O movimento se dá início com a palma da mão para cima, realizando a flexão do antebraço completamente e retornando à posição inicial da extensão do antebraço. $\mathrm{O}$ avaliador deve manter-se junto ao participante, colocando os dedos no bíceps de forma a estabilizar o movimento. Pontuação: O número máximo de execuções realizadas nos 30 segundos (Rickli \& Jones C.J, 2008).

\section{Teste de velocidade de marcha de 6 minutos}

O participante deve percorrer a maior distância possível caminhando no período de seis minutos. Pontuação: Marcar a distância máxima que percorreu durante os seis minutos (Enright et al., 2003).

A Figura 1 demonstra o fluxo de avaliações realizadas no período pré e pós intervenção.

Figura 1. Desenho experimental do protocolo utilizado.

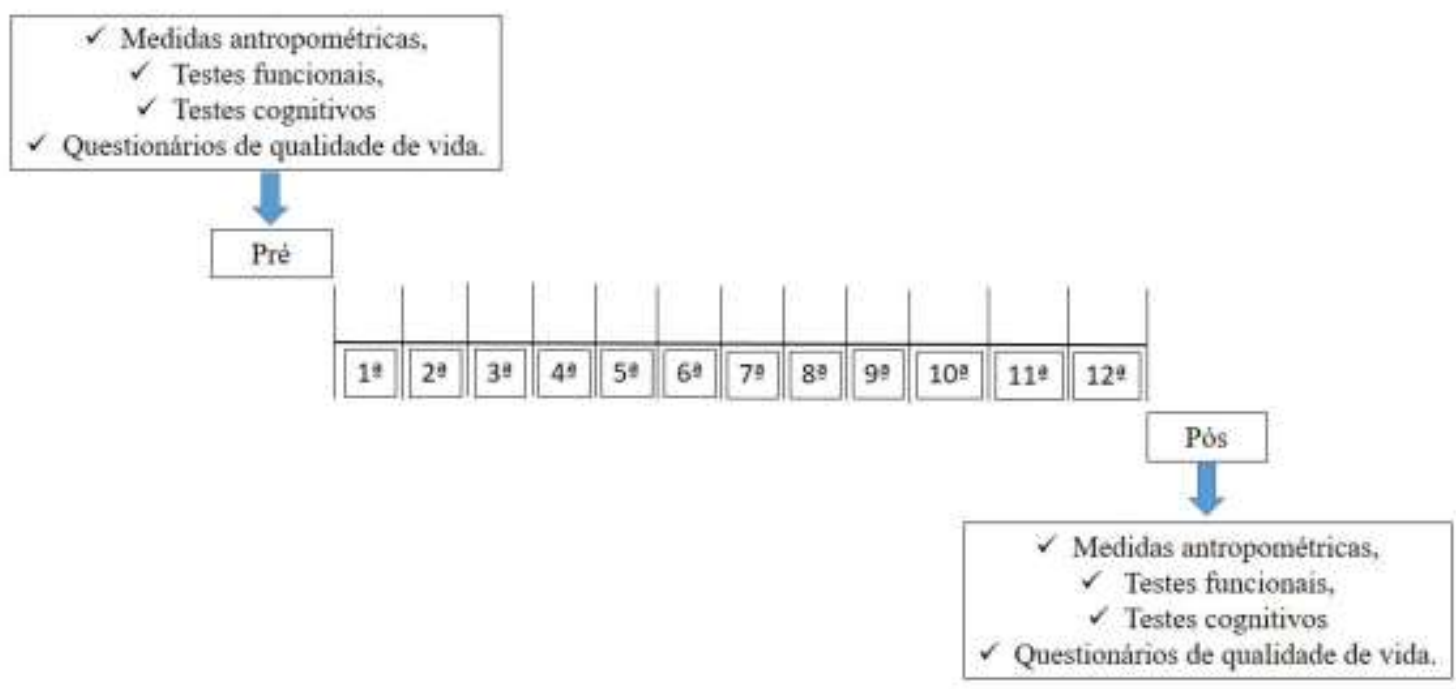

Fonte: Autores. 


\section{Análise Estatística}

Para a análise estatística utilizou-se o software PRISMA versão 6.0. A normalidade dos dados foi verificada pelo Teste de Shapiro-Wilk. Utilizou-se o teste t-student para comparação das variáveis entre os períodos pré e pós-intervenção. Os dados foram apresentados como média, desvio padrão e o intervalo de confiança de $95 \%$. O nível de significância adotado foi de $\alpha \leq 0,05$.

\section{Resultados}

A Tabela 2 apresenta as variáveis descritivas da amostra no período Pré-intervenção.

Tabela 2. Variáveis descritivas da amostra no período pré-intervenção.

\begin{tabular}{lll}
\hline VARIÁVEL & \multicolumn{2}{l}{ PRÉ-INTERVENÇÃO } \\
\hline & MÉDIA & DP \\
Idade (anos) & 68,5 & 6,95 \\
Massa corporal Total (MCT) (Kg) & 66,73 & 14,59 \\
Estatura (m) & 1,53 & 0,1 \\
IMC (MCT/Estatura ${ }^{2}$ ) & 28,2 & 5,29 \\
\hline
\end{tabular}

Dados apresentados em média e desvio padrão (DP). IMC: índice de massa corporal. Fonte: Autores.

A Figura 2 demonstra os resultados relativos ao questionário de qualidade de vida nos períodos pré e pós treinamento

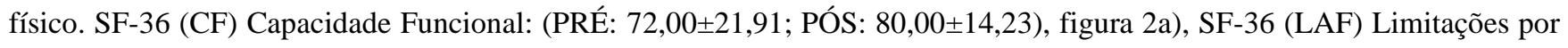

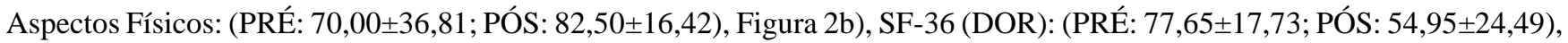

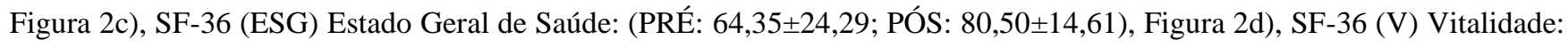

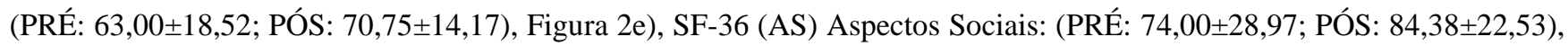

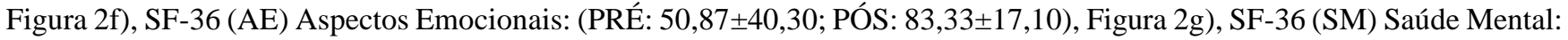

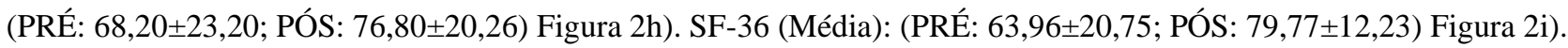


Figura 2. Questionário de qualidade de vida.

a)
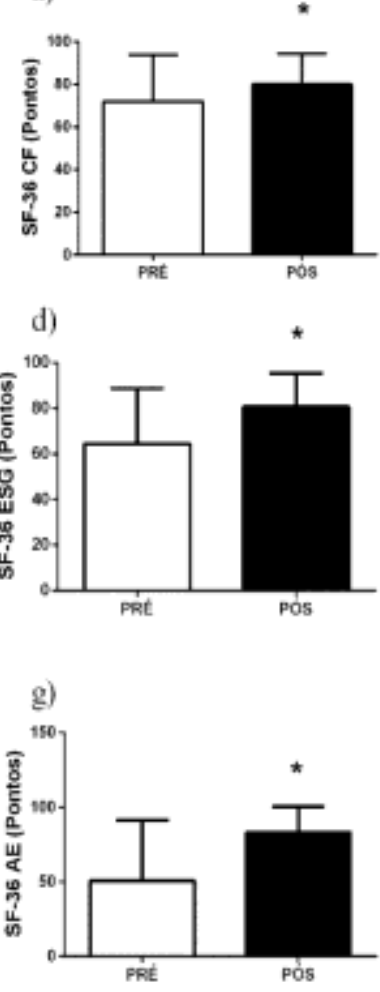

b)

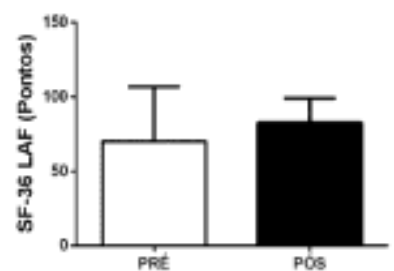

e)

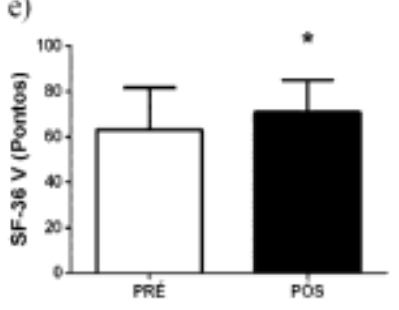

h)

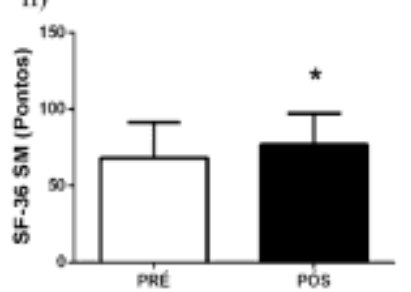

c)

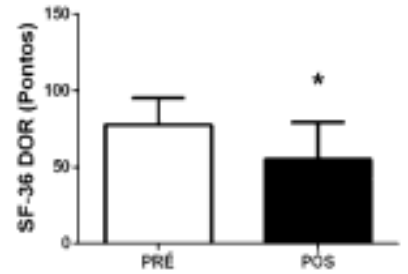

f)
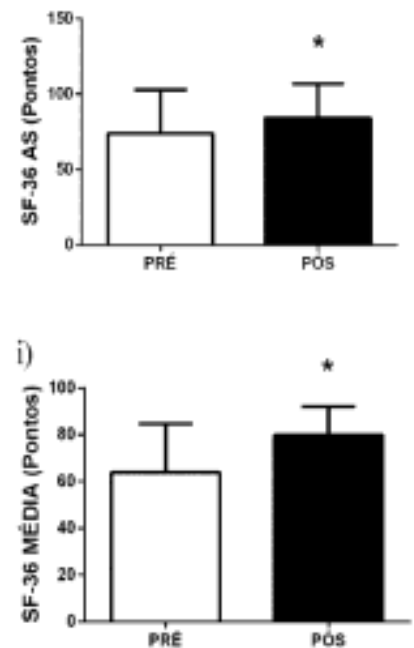

Dados apresentados em média e desvio padrão (DP). a) SF-36 (CF), b) SF-36 (LAF), c) SF-36 (DOR), d) SF-36 (ESG), e) SF-36 (V), f) SF-36 (AS), g) SF-36 (AE), h) SF-36 (SM), i) SF-36 (Média). Teste t-Student pareado, n=20. *(p<0,05). Fonte: Autores.

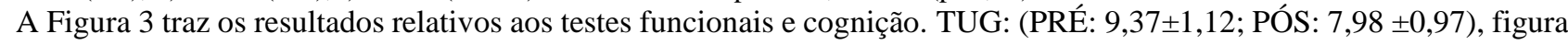

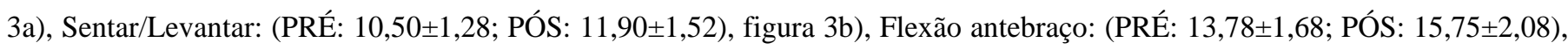

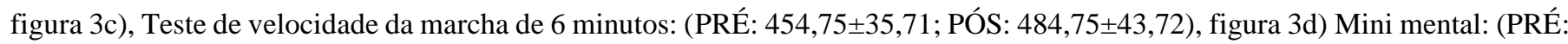

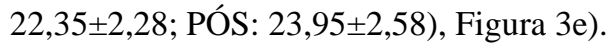


Figura 3. Testes funcionais e cognição.

a)

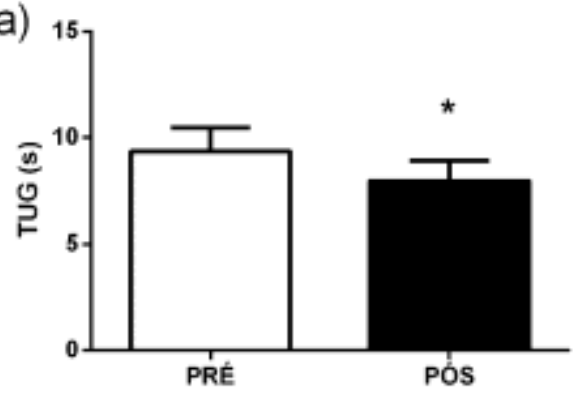

c)

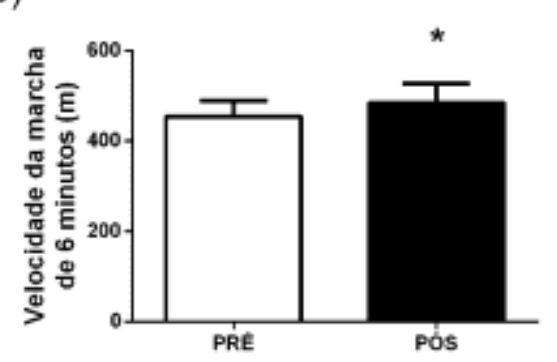

b)

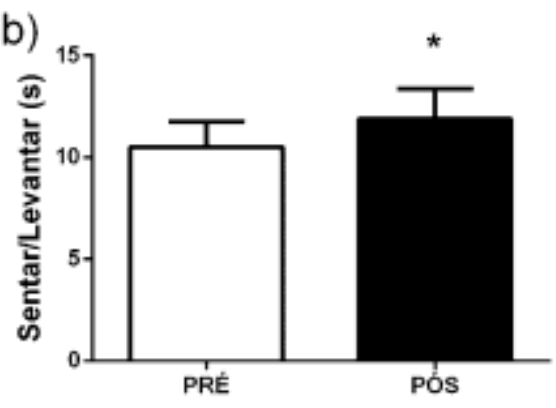

d)

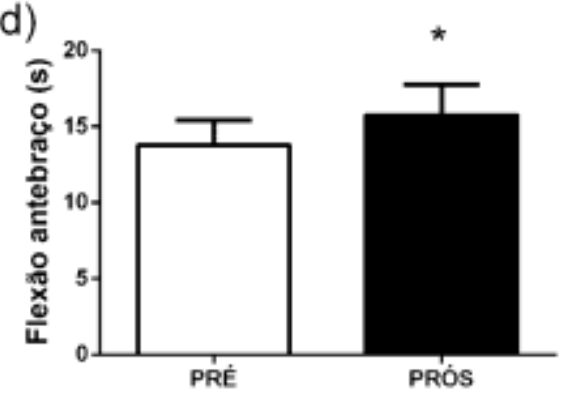

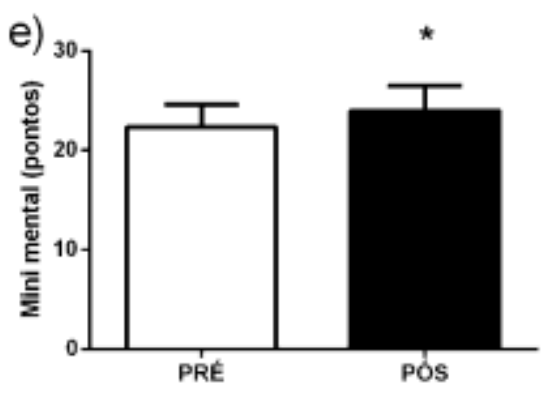

Dados apresentados em média e desvio padrão (DP). a) TUG, b) Sentar/Levantar, c) Flexão antebraço, d) Velocidade da marcha de 6 minutos, e) Mini mental. Teste t-Student pareado, $n=20 . *(p<0,05)$. Fonte: Autores.

\section{Discussão}

Nesse estudo avaliaram-se os efeitos de 12 semanas de um programa de treinamento físico em circuito sobre aspectos cognitivos, funcionais e qualidade de vida de idosos. Os resultados obtidos demonstram a efetividade do protocolo proposto, por meio do impacto positivo do exercício sobre esses parâmetros após a realização do protocolo proposto.

Foram utilizados testes que são amplamente realizados para avaliação funcional de idosos, tais como o teste de velocidade de marcha de 6 minutos, teste de sentar e levantar, teste de flexão de antebraço e teste Time Up and Go (Coelho-Junior et al., 2018). Avaliação essa que foi acrescida pelo mini exame de estado mental, que permite a avaliação da função cognitiva e rastreamento de quadros demenciais, e pode ser utilizado isoladamente, ou como no presente estudo incorporado a outros instrumentos de avaliação como o SF-36, utilizado para avaliar a qualidade de vida (Lourenço \& Veras, 2006).

No estudo de Dias e colaboradores (2014) 104 idosos realizavam aulas de ginástica funcional, com intensidade moderada, três vezes por semana durante 60 minutos por sessão, em um período de 6 meses e obtiveram melhoras nas capacidades cognitivas quando avaliados pelo MEEM. Os dados obtidos no presente estudo demonstram melhora significativa na pontuação obtida pelos participantes ao realizarem o MEEM, uma vez que os resultados anteriores ao período de intervenção demonstram uma média de 22,35 pontos, o que já sugeriria um quadro de demência. No entanto, após 12 semanas de intervenção, a média passou para 23,95 pontos, saindo da margem sugestiva para demência. Evidências apontam que um comprometimento cognitivo leve é comum na 
população idosa e esse declínio da capacidade cognitiva é consequência do processo fisiológico de envelhecimento ou de um estágio de transição para as demências (Da Costa et al., 2015).

O baixo grau de escolaridade deve ser levado em conta no que diz respeito aos resultados obtidos no presente estudo para os testes cognitivos (Ribeiro et al., 2019), todavia o protocolo de exercício desenvolvido nesse cenário fez com que os idosos saíssem de um estágio prévio de demência para um grau de normalidade, sendo imprescindível destacar que a demência é um problema de saúde mental que cresce exponencialmente em todo o mundo, e no Brasil não é diferente, acarretando no despenho financeiro de milhões de reais e o inestimável custo da perda da qualidade de vida da população, sobretudo para indivíduos após os 60 anos (Rosa et al., 2018).

(Liposcki et al., 2019), desenvolveram um programa de treinamento físico 2 vezes por semana durante 6 meses e encontraram melhora da qualidade de vida em idosas. O estudo conduzido por (Mota et al., 2006), avaliou efeitos do exercício físico sobre a qualidade de vida de idosos utilizando o SF-36 após a realização de um programa de exercícios físicos duas vezes por semana durante 40 semanas. Foram observadas melhoras significativas na qualidade de vida, resultado compatível com o presente estudo, com o diferencial que a intervenção aqui proposta foi realizada em um período de 12 semanas.

O exercício físico é reconhecidamente uma ferramenta não farmacológica eficiente no que diz respeito a promoção da saúde mental, qualidade de vida e cognição em idosos (Bento-Torres et al., 2019; Mendes \& Novelli, 2015), devendo-se levar em conta que são encontrados maiores indicies de indivíduos acometidos por patologias de ordem mental a medida que se aumenta a expectativa de vida, sobretudo quando esse aumento na expectativa de vida é acompanhado pelo sedentarismo (Willey et al., 2016).

No estudo de (Fernandes et al., 2012) foi desenvolvido um programa de treinamento durante 6 meses com idosos e observou-se reduções significativas no tempo de realização do teste de TUG. Resultados similares aos encontrados no presente estudo, com o diferencial que o protocolo aqui proposto em apenas 50\% do tempo utilizado por Fernandes e colaboradores (Fernandes et al., 2012). Sendo demonstrada na literatura pregressa que melhores resultados no TUG possivelmente se relacionam com menor incidência de quedas, em idosos, explicitando que o aumento da capacidade funcional deve ser considerado como um fator de promoção da saúde (Silva et al., 2016).

(Alves et al., 2004) realizaram um estudo com 74 mulheres idosas que praticaram hidroginástica durante 12 semanas e encontraram melhorias significativas no teste de flexão de antebraço, ou seja, foi demonstrada a eficiência do exercício em aumentar a força e resistência dos membros superiores. (Parra et al., 2009) encontraram melhora no mesmo teste em idosas após uma intervenção de 12 semanas de treinamento com exercício resistido. Os dados apresentados corroboram com os achados do presente estudo, e reforçam importância do exercício físico para ganho e manutenção da força de membros superiores independente da modalidade adotada. A força de membros superiores ainda pode se correlacionar com o nível de independência funcional em idosos, tendo em vista que a mesma é muito requisitada durante as Atividades da Vida Diária (AVDs) (da Cunha \& Gress, 2012).

(Cao et al., 2007) demonstraram que um programa de treinamento de 12 semanas foi suficiente para uma melhora na força de membros inferiores. Resultados que vão de encontro aos achados do presente estudo diante da pronunciada melhoria encontrada no teste de caminhada de 6 minutos. (Garcia et al., 2011) demonstraram que a força e potência muscular apresentam correlações moderadas com a velocidade de marcha habitual (VMH) e a velocidade de marcha máxima (VMM). Sendo assim, existe a relação que sugere que o programa de treinamento seja capaz de aumentar a força de membros inferiores bem como a velocidade de marcha e consequentemente reduzir o risco de queda em idosos.

Foi explicitado em trabalhos anteriores que existe uma relação diretamente proporcional entre perda da capacidade motora e aumento de distúrbios como demência (Hackett et al., 2018; Middleton et al., 2015). Diante disso, intervenções como o exercício físico mostram-se fundamentais por prevenir ou mesmo retardar esse declínio funcional que incide em diversos parâmetros relacionados à saúde (de Moraes et al., 2016; Tse et al., 2018), além da relação existente entre dificuldade da marcha e fragilidade em idosos (Puts et al., 2017). 
Já foi demonstrada a efetividade do exercício físico no que diz respeito à promoção da saúde em populações idosas (Da Costa et al., 2015), todavia é comum encontrar trabalhos que sejam realizados por um período prolongado de tempo, por mais de 12 semanas e que utilizaram metodologias predominantemente aeróbicas ou resistidas, sendo escassas referências que tenham utilizado um período reduzido de intervenção e a metodologia de circuito. Além do fato de muitas vezes os trabalhos realizados utilizando-se exercícios físicos se pautarem unicamente sobre a premissa do aumento do desempenho por meio do treinamento, no entanto o protocolo desenvolvido no presente estudo contava com atividades lúdicas durante o período de aquecimento, contribuindo assim para a melhoria da autoestima, redução de fatores estressantes, estimulando a convivência social, além de questões de ordem psíquicas e cognitivas (Guimarães et al., 2016).

Classicamente são propostos exercícios resistidos para idosos quando se pretende alcançar ganhos de força muscular e exercícios aeróbicos com o intuito de galgar ganhos no sistema cardiovascular (Bueno et al., 2018; Krause Neto et al., 2018; Oliveira et al., 2018; Scianni et al., 2019), porém são escassos os estudos que se valem das metodologias de treinamento de forma combinada em circuito por um período reduzido de tempo.

Os resultados do presente estudo demonstram a eficiência de um protocolo de curto período de aplicação, que se valeu do adendo da realização de atividades lúdicas, tendo como resultado adaptações positivas significativas sobre funcionalidade, cognição e qualidade de vida de indivíduos idosos, achados que vão de encontro a políticas públicas de promoção de saúde, tendo em vista o incremento da população idosa no Brasil. Obviamente que são necessários mais estudos com o objetivo de gerar maior base teórica sobre a temática, todavia a metodologia desenvolvida demonstra promissoras expectativas.

\section{Conclusão}

O programa de exercícios físicos proposto foi capaz de trazer melhorias significativas sobre a funcionalidade, cognição e qualidade de vida em idosos. Podendo servir como uma alternativa eficiente na promoção da saúde para essa população. Com destaque para o baixo despenho financeiro da metodologia, o que pode facilitar sua aplicação pelo Sistema Único de Saúde (SUS) brasileiro.

Por fim assumimos que o trabalho possui limitações por trabalhar com uma amostra por conveniência e heterogêneo, para os próximos estudos é necessário aumentar a amostra e definir de melhor forma o público, investigar as vias metabólicas que são afetadas pelo exercício proposto, além da utilização de outros métodos de treinamento para avaliar a eficiência do treinamento.

\section{Referências}

Alves, R. V., Mota, J., Costa, M. da C., \& Alves, J. G. B. (2004). Aptidão física relacionada à saúde de idosos: influência da hidroginástica. Revista Brasileira de Medicina Do Esporte, 10(1), 31-37. https://doi.org/10.1590/s1517-86922004000100003

Bento-Torres, N. V. O., Bento-Torres, J., Tomás, A. M., de Souza, L. G. T., De Freitas, J. O., Pantoja, J. A. dos S., \& Picanço-Diniz, C. W. (2019). Hidroginástica E Treinamento Resistido Melhoram a Cognição De Idosos. Revista Brasileira de Medicina Do Esporte, 25(1), 71-75. https://doi.org/10.1590/1517869220192501190627

Bohannon, R. W., \& Schaubert, K. (2006). Long-term reliability of the timed up-and-go test among community-dwelling elders. Journal of Physical Therapy Science, 17(2), 93-96. https://doi.org/10.1589/jpts.17.93

Borg, G. (1998). Borg's Perceived Exertion and Pain Scales (B. Lane \& J. Rhoda (eds.)). Human Kinetics.

Brandão, G. S., Oliveira, L. V. F., Brandão, G. S., Silva, A. S., Sampaio, A. A. C., Urbano, J. J., Soares, A., Santos Faria, N., Pasqualotto, L. T., Oliveira, E. F., Oliveira, R. F., Pires-Oliveira, D. A. A., \& Camelier, A. A. (2018). Effect of a home-based exercise program on functional mobility and quality of life in elderly people: Protocol of a single-blind, randomized controlled trial. Trials, 19(1), 1-10. https://doi.org/10.1186/s13063-018-3061-1

Bueno, G. A. S., Menezes, R. L. de, Vilela Lemos, T., \& Gervásio, F. M. (2018). Relationship of muscle strength with static balance in elderly - comparison between pilates and multimodalities. Revista Brasileira de Ciencias Do Esporte, 40(4), 435-441. https://doi.org/10.1016/j.rbce.2018.04.008

Campolina, A. G., Bortoluzzo, A. B., Ferraz, M. B., \& Ciconelli, R. M. (2011). Validação da versão brasileira do questionário genérico de qualidade de vida shortform 6 dimensions (SF-6D Brasil). Ciencia e Saude Coletiva, 16(7), 3103-3110. https://doi.org/10.1590/s1413-81232011000800010

Cao, Z. B., Maeda, A., Shima, N., Kurata, H., \& Nishizono, H. (2007). The effect of a 12-week combined exercise intervention program on physical performance 
and gait kinematics in community-dwelling elderly women. Journal of Physiological Anthropology, 26(3), 325-332. https://doi.org/10.2114/jpa2.26.325

Cassilhas, R. C., Tufik, S., Antunes, H. K. M., \& de Mello, M. T. (2010). Mood, anxiety, and serum IGF-1 in elderly men given 24 weeks of high resistance exercise. Perceptual and Motor Skills, 110(1), 265-276. https://doi.org/10.2466/PMS.110.1.265-276

Cassilhas, R. C., Viana, V. A. R., Grassmann, V., Santos, R. T., Santos, R. F., Tufik, S., \& Mello, M. T. (2007). The impact of resistance exercise on the cognitive function of the elderly. Medicine and Science in Sports and Exercise, 39(8), 1401-1407. https://doi.org/10.1249/mss.0b013e318060111f

Coelho-Junior, H. J., Rodrigues, B., Gonçalves, I. de O., Asano, R. Y., Uchida, M. C., \& Marzetti, E. (2018). The physical capabilities underlying timed "Up and Go" test are time-dependent in community-dwelling older women. Experimental Gerontology, 104(September 2017), 138-146. https://doi.org/10.1016/j.exger.2018.01.025

Copeland, J. L., Good, J., \& Dogra, S. (2018). Strength training is associated with better functional fitness and perceived healthy aging among physically active older adults: a cross-sectional analysis of the Canadian Longitudinal Study on Aging. Aging Clinical and Experimental Research, 31(9), 1257-1263. https://doi.org/10.1007/s40520-018-1079-6

Da Costa, L. da S. V., Moreira de Sousa, N., Geraldo Alves, A., Vargas de Brito Alves, F. A., Ferraz Araújo, R., \& Santos Nogueira, M. (2015). Análise Comparativa Da Qualidade De Vida, Equilíbrio E Força Muscular Em Idosos Praticantes De Exercício Físico E Sedentários 1 Comparative Analysis of Quality of Life Balance and Muscle Strength in Elderly Individuals Practicing Physical Exercise and Sed. Revista Faculdade Montes Belos, 8(3), 61-179. https://doi.org/10.5335/rbceh.2010.055

da Cunha, R. L., \& Gress, F. A. G. (2012). Resultados Dos Níveis De Capacidades Físicas De Idosas Praticantes De Um Programa De Exercícios Físicos Com Frequência De De 1 Vez Por Semana. Rev. Acta Brasileira Do Movimento Humano, 2(3), 14-31. https://doi.org/10.1017/CBO9781107415324.004

de Melo, D. M., \& Barbosa, A. J. G. (2015). O uso do Mini-Exame do Estado Mental em pesquisas com idosos no Brasil: Uma revisão sistemática. Ciencia e Saude Coletiva, 20(12), 3865-3876. https://doi.org/10.1590/1413-812320152012.06032015

de Moraes, E. N., do Carmo, J. A., de Moraes, F. L., Azevedo, R. S., Machado, C. J., \& Montilla, D. E. R. (2016). Clinical-Functional Vulnerability Index-20 (IVCF-20): Rapid recognition of frail older adults. Revista de Saude Publica, 50, 1-9. https://doi.org/10.1590/S1518-8787.2016050006963

Enright, P. L., McBurnie, M. A., Bittner, V., Tracy, R. P., McNamara, R., Arnold, A., \& Newman, A. B. (2003). The 6-min walk test: A quick measure of functional status in elderly adults. Chest, 123(2), 387-398. https://doi.org/10.1378/chest.123.2.387

Fernandes, A. M. B. L., Ferreira, J. J. de A., Stolt, L. R. O. G., Brito, G. E. G. de, Clementino, A. C. C. R., \& Sousa, N. M. de. (2012). Efeitos da prática de exercício físico sobre o desempenho da marcha e da mobilidade funcional em idosos. Fisioterapia Em Movimento, 25(4), 821-830. https://doi.org/10.1590/s010351502012000400015

Galloza, J., Castillo, B., \& Micheo, W. (2017). Benefits of Exercise in the Older Population. Physical Medicine and Rehabilitation Clinics of North America, 28(4), 659-669. https://doi.org/10.1016/j.pmr.2017.06.001

Garcia, P. A., Dias, J. M. D., Dias, R. C., Santos, P., \& Zampa, C. C. (2011). Estudo da relação entre função muscular, mobilidade funcional e nível de atividade física em idosos comunitários. Revista Brasileira de Fisioterapia, 15(1), 15-22.

Guimarães, A., Dutra, N., Silva, G., Vieira-Silva, M., \& Maia, B. (2016). Atividades grupais com idosos institucionalizados: exercícios físicos funcionais e lúdicos em ação transdisciplinar. Pesquisas e Práticas Psicossociais, 11(2), 443-452.

Hackett, R. A., Davies-Kershaw, H., Cadar, D., Orrell, M., \& Steptoe, A. (2018). Walking Speed, Cognitive Function, and Dementia Risk in the English Longitudinal Study of Ageing. Journal of the American Geriatrics Society, 66(9), 1670-1675. https://doi.org/10.1111/jgs.15312

IBGE. (2018). Numero de idosos cresce $18 \%$ em 5 anos e ultrapassa 30 milhoes em 2017. https://agenciadenoticias.ibge.gov.br/agencia-noticias/2012-agencia-denoticias/noticias/20980-numero-de-idosos-cresce-18-em-5-anos-e-ultrapassa-30-milhoes-em-2017

Krause Neto, W., de Assis Silva, W., Polican Ciena, A., Bocalini, D., Aparecido Baptista Nucci, R., Alberto Anaruma, C., \& Florencio Gama, E. (2018). Total training load may explain similar strength gains and muscle hypertrophy seen in aged rats submitted to resistance training and anabolic steroids. Aging Male, 21(1), 65-76. https://doi.org/10.1080/13685538.2017.1365832

Lacroix, A., Kressig, R. W., Muehlbauer, T., Gschwind, Y. J., Pfenninger, B., Bruegger, O., \& Granacher, U. (2016). Effects of a supervised versus an unsupervised combined balance and strength training program on balance and muscle power in healthy older adults: A randomized controlled trial. Gerontology, 62(3), 275288. https://doi.org/10.1159/000442087

Liposcki, D. B., da Silva Nagata, I. F., Silvano, G. A., Zanella, K., \& Schneider, R. H. (2019). Influence of a Pilates exercise program on the quality of life of sedentary elderly people: A randomized clinical trial. Journal of Bodywork and Movement Therapies, 23(2), 390-393. https://doi.org/10.1016/j.jbmt.2018.02.007

Lira, V. A., Gil, C., \& Araújo, S. De. (2000). ARTIGO ORIGINAL Teste de sentar-levantar: estudos de fidedignidade Sitting-rising test: reliability studies. Medicina, 09-18.

Lopez, P., Pinto, R. S., Radaelli, R., Rech, A., Grazioli, R., Izquierdo, M., \& Cadore, E. L. (2018). Benefits of resistance training in physically frail elderly: a systematic review. Aging Clinical and Experimental Research, 30(8), 889-899. https://doi.org/10.1007/s40520-017-0863-z

Lourenço, R. A., \& Veras, R. P. (2006). Mini-Exame do Estado Mental: características psicométricas em idosos ambulatoriais. Revista de Saúde Pública, 40(4), 712-719. https://doi.org/10.1590/s0034-89102006000500023

Mendes, R. S., \& Novelli, M. M. P. C. (2015). Perfil Cognitivo E Funcional De Idosos Moradores De Uma Instituição De Longa Permanência Para Idosos. Cadernos de Terapia Ocupacional Da UFSCar, 23(4), 723-731. https://doi.org/10.4322/0104-4931.ctoao0535

Middleton, A., Fritz, S. L., \& Lusardi, M. (2015). Walking speed: The functional vital sign. Journal of Aging and Physical Activity, 23(2), 314-322. 
Research, Society and Development, v. 10, n. 9, e13610917091, 2021

(CC BY 4.0) | ISSN 2525-3409 | DOI: http://dx.doi.org/10.33448/rsd-v10i9.17091

https://doi.org/10.1123/japa.2013-0236

Mota, J., Ribeiro, J. L., Carvalho, J., \& Matos, M. G. de. (2006). Atividade física e qualidade de vida associada à saúde em idosos participantes e não participantes em programas regulares de atividade física. Rev. Bras. Educ. Fís. Esp, January, 219-225. https://doi.org/10.1590/S1807-55092006000300007

Oliveira, D. V. de, Nascimento Júnior, J. R. A. do, Moreira, C. R., Bertolini, S. M. M. G., Prati, A. R. C., \& Cavaglieri, C. R. (2018). Factors associated with body image dissatisfaction of the elderly who practice weight training. Fisioterapia Em Movimento, 31(0), 1-10. https://doi.org/10.1590/1980-5918.031.ao13

Parra, M. T., Parra, S. A., Oliveira, A. R., \& Greguol, M. (2009). Influência Do Treinamento Com Pesos Sobre a Capacidade Funcional De Mulheres Idosas. Revista Brasileira de Ciência e Movimento, 17(3), 32-38. https://doi.org/10.18511/rbcm.v17i3.956

Pereira, A., Shitsuka, D., Parreira, F., \& Shitsuka, R. (2018). Método Qualitativo, Quantitativo ou Quali-Quanti. In Metodologia da Pesquisa Científica. https://repositorio.ufsm.br/bitstream/handle/1/15824/Lic_Computacao_Metodologia-Pesquisa-Cientifica.pdf?sequence=1. Acesso em: 28 março 2020.

Pietta-Dias, C., Bello, M. D., da Silva, R., Vargas, C., Machado, G. D. B., Roncada, C., Tiggemann, C. L., \& Schröder, N. (2019). Differential impact of endurance, strength, or combined training on quality of life and plasma serotonin in healthy older women. Aging Clinical and Experimental Research, 31(11), 1573-1581. https://doi.org/10.1007/s40520-019-01120-x

Puts, M. T. E., Toubasi, S., Andrew, M. K., Ashe, M. C., Ploeg, J., Atkinson, E., Ayala, A. P., Roy, A., Rodríguez Monforte, M., Bergman, H., \& McGilton, K. (2017). Interventions to prevent or reduce the level of frailty in community-dwelling older adults: a scoping review of the literature and international policies. Age and Ageing. https://doi.org/10.1093/ageing/afw247

Raso, V., Cassilhas, R. C., Santana, M. G. de, Boscolo, R. A., Viana, V. A. R., Grassmann, V., Tufik, S., \& Mello, M. T. de. (2016). Predictors of muscle strength in older individuals. Medical Express, 3(3), 1-8. https://doi.org/10.5935/medicalexpress.2016.03.09

Ribeiro, I. A., Lima, L. R. de, Volpe, C. R. G., Funghetto, S. S., Rehem, T. C. M. S. B., \& Stival, M. M. (2019). Síndrome do idoso frágil em idosos com doenças crônicas na Atenção Primária. Rev Esc Enferm USP, 53(03449), 1-9.

Rickli, R. ., \& Jones C.J. (2008). Teste de aptidão física para idosos. Manole.

Rosa, T. S. M., Dos Santos Filha, V. A. V., \& de Moraes, A. B. (2018). Prevalence and factors associated with cognitive impairments in the elderly of charity asylums: A descriptive study. Ciencia e Saude Coletiva, 23(11), 3757-3765. https://doi.org/10.1590/1413-812320182311.25212016

Scianni, A. A., Faria, G. S. e., Silva, J. S. da, Benfica, P. do A., \& Faria, C. D. C. de M. (2019). Effects of physical exercises on the nervous system of elders and its functional consequences. Revista Brasileira de Ciencias Do Esporte, 41(1), 81-95. https://doi.org/10.1016/j.rbce.2018.03.026

Silva, C. D. A. da, Tarcisia, S., Ferreira, R., Claudia, M., Alves, C. H., Virginia, K., \& Soares, B. D. C. (2016). Avaliação do risco de queda e da demanda atencional em idosos submetidos a um protocolo cinesioterapêtico de dupla tarefa. 17-25.

Tse, M. M. Y., Kwan, R. Y. C., \& Lau, J. L. (2018). Ageing in individuals with intellectual disability: Issues and concerns in Hong Kong. Hong Kong Medical Journal, 24(1), 68-72. https://doi.org/10.12809/hkmj166302

Willey, J. Z., Gardener, H., Caunca, M. R., Moon, Y. P., Dong, C., Cheung, Y. K., Sacco, R. L., Elkind, M. S. V., \& Wright, C. B. (2016). Leisure-time physical activity associates with cognitive decline. Neurology, 86(20), 1897-1903. https://doi.org/10.1212/WNL.0000000000002582 